\title{
Efficient Orthogonal Bicomplex Bilinear DSP Algorithm Design
}

\author{
Zlatka Valkova-Jarvis* (Associate Professor, Department of Communication Networks, Faculty of \\ Telecommunications, Technical University of Sofia, Sofia, Bulgaria), \\ Dimitriya Mihaylova (Engineer, Technical University of Sofia, Sofia, Bulgaria), \\ Viktor Stoynov (Engineer, Technical University of Sofia, Sofia, Bulgaria)
}

\begin{abstract}
The present paper describes the development of a new technique for designing orthogonal bicomplex Digital Signal Processing (DSP) algorithms. In contrast to those previously reported on, this novel method is of universal application while being unaffected by either the type or the order of the real digital processing algorithm employed as a prototype. The proposed technique builds on Watanabe and Nishihara's complex orthogonal transformation, and converts real or complex orthogonal transfer functions into bicomplex orthogonal ones. In this study, the new technique is applied to the design and testing of orthogonal bilinear bicomplex filters with a canonical number of elements, the main advantage of which is that they are several times lower in order. In this way, bilinear bicomplex orthogonal transfer functions are made up of real coefficient ones of the fourth-order, thereby reducing the order of the filter by a factor of four. The experiments demonstrate that the properties of the prototype filter are acquired by the bicomplex orthogonal filters, irrespective of the prototype being complex or real in nature.
\end{abstract}

Keywords - Bicomplex digital filters; Orthogonal complex digital filter; Sensitivity; Word-length.

\section{INTRODUCTION}

Hypercomplex numbers are an expansion of complex numbers. Hypercomplex arithmetic is based on quaternions and biquaternions, a concept developed by the Irish mathematician Hamilton in 1843 [1]. Later Schutte and Wenzel [2] determined that Hamilton's number system is not well suited for use in Digital Signal Processing (DSP) and in 1990 they introduced a different number system, named Reduced Biquaternions (RBs), also known as bicomplex numbers. This latter terminology will be utilized from here on in this paper. Thenceforward bicomplex arithmetic has been applied in a number of areas, such as image processing, computer graphics, aerospace engineering, etc.

Modern-day telecommunications often employ narrowband signals, which are complex in nature and are best processed through the use of complex DSP algorithms. Orthogonal complex filters [3] now represent a well-developed field, both theoretically and experimentally, and they are therefore used in the processing of a special class of narrowband signals, termed analytical signals. Hypercomplex signals as an extension of complex signals has already been introduced [4] and a large number of research studies relating to their processing have been reported. Digital filters with bicomplex coefficients are widely applicable digital processing algorithms and are used in many applications such as colour-based object and image recognition [5]-[7], smoothing colour image components [8], hash authentication of images [9], image processing of both colour and grey scale images [10], auto- and cross-correlation of colour image processing [11], [12], and multispectral recognition [13].

Both computational efficiency and the stability criterion of digital filters with bicomplex coefficients have also been investigated [14], [15]. Bicomplex digital filters significantly reduce the filter order - by twice the order for a complex filter and four times the order for a real coefficient filter - which is their main advantage. Numerous approaches to designing bicomplex filters have been put forward in the literature [16][19], most of them having been developed for specific types of digital filters and thus rendering them non-universal. Orthogonal transforms, for example, the Quaternion Polar Harmonic Transform (QPHT) [20] and Quaternion Polar Linear Canonical Transform (QPLCT) [21], are employed in the improvement of image representation capability and numerical stability. In [22] and [23], a design of orthogonal filters with hypercomplex coefficients, based on the concept that "an orthogonal filter with real coefficients is expanded into one with hypercomplex coefficients", is proposed. This design method is based on real all-pass IIR filter sections "through the use of an orthogonal polynomial expansion", which does not result in a bicomplex structure capable of processing a pair of orthogonal complex signals.

This publication describes the development of a new design procedure for orthogonal bicomplex DSP algorithms. The procedure is applied to derive orthogonal bicomplex bilinear IIR digital filters, and is suitable for any filter structure of any order. As many DSP applications require efficient high accuracy filters with very short word-length coefficients that can be achieved by using low-sensitivity structures, a bilinear narrow-band bicomplex digital filter with very low coefficient

\footnotetext{
${ }^{*}$ Corresponding author.

E-mail: zvv@tu-sofia.bg
} 
sensitivity is designed here, using the proposed new technique, and a comparative study is undertaken.

The paper is arranged in the following way: Section II outlines the main properties of bicomplex numbers. In Section III, a new design procedure for orthogonal bicomplex digital filters is presented. Bilinear bicomplex orthogonal filters are derived in Section IV and their sensitivity is experimentally investigated in Section V. Section VI concludes the paper.

\section{PROPERTIES OF BICOMPLEX NUMBERS}

A quaternion may be represented in hypercomplex form as follows:

$$
q=q_{1}+i q_{2}+j q_{3}+k q_{4},
$$

where $q_{1}, q_{2}, q_{3}$ and $q_{4}$ are real numbers, while $i, j$, and $k$ are orthogonal complex operators that obey the following rules:

$$
\begin{gathered}
i^{2}=j^{2}=k^{2}=i j k=-1 ; \\
i j=k ; j k=i ; k i=j ; j i=-k ; k j=-i ; i k=-j .
\end{gathered}
$$

In respect of the arithmetic laws of a quaternion, addition of two quaternions is commutative and associative, while multiplication is associative but not commutative, which renders them inapplicable to DSP systems.

In order to solve this problem, bicomplex numbers derived as follows have been proposed: $q_{3}$ and $q_{4}$ are set to zero and $q_{1}$ and $q_{2}$ are expanded to complex numbers. This modification is equivalent to the expansion of each element of a complex number so that it becomes a complex number itself hence, its name - bicomplex number:

$$
\begin{aligned}
a & =a_{1}+i a_{2}=\left[A_{1}+j A_{2}\right]+i\left[A_{3}+j A_{4}\right]= \\
& =A_{S}+i A_{V}=A_{1}+j A_{2}+i A_{3}+k A_{4}=A,
\end{aligned}
$$

where $A_{1}, A_{2}, A_{3}$ and $A_{4}$ are real numbers, $j$ is the imaginary unit with $j^{2}=-1$ and $i$ is the vector unit with $i^{2}=-1$. The complex numbers $A_{S}$ and $A_{V}$ are called the scalar part and vector part, respectively. The properties of the imaginary units of a bicomplex number $A$ are as follows [2]:

$$
\begin{gathered}
i^{2}=j^{2}=-1 ; \quad k^{2}=1 ; \\
i j=j i=k ; \quad j k=k j=-i ; \quad k i=k i=-j .
\end{gathered}
$$

Two types of conjugate of $A$ can be defined - the vector conjugate:

$$
A^{+}=A_{S}-i A_{V}=\left(A_{1}+j A_{2}\right)-i\left(A_{3}+j A_{4}\right)
$$

and the complex conjugate:

$$
A^{*}=A_{S}^{*}+i A_{V}^{*}=\left(A_{1}-j A_{2}\right)+i\left(A_{3}-j A_{4}\right) .
$$

Moreover, a double, or hypercomplex, conjugate can also be defined as:

$$
A^{*+}=A_{S}^{*}-i A_{V}^{*}=\left(A_{1}-j A_{2}\right)-i\left(A_{3}-j A_{4}\right) .
$$

The norm of a bicomplex number is defined in the range of complex numbers and is as follows:

$$
N(A)=A A^{+}=\left(A_{S}+i A_{V}\right)\left(A_{S}-i A_{V}\right) .
$$

Applying Euler's formula for the complex exponential, generalized to a hypercomplex form, any quaternion q may be represented in polar form as:

$$
q=|q| e^{\mu \Phi}
$$

$\mu$ and $\Phi$ are referred to as the eigenaxis and eigenangle of $q$, respectively. $\Phi$ is analogous to the argument of a complex number, but is unique only in the range $[0, \pi]$ because any value greater than $\pi$ can be reduced to this range by reversing the eigenaxis. $\mu$ is a pure quaternion and identifies the direction in three-space of the hypercomplex number's vector part. The requirement for $\mu$ is $|\mu|=1$. In case of orthogonality the eigenangle $\Phi$ is equal to $\pi / 2$.

From here on in this publication each input, output or internal signal and filter coefficient will be encoded as a real (R), complex (C) or bicomplex (biC) number.

\section{BiCOMPLEX ORTHOgONAL Digital FILTER DESIGN PROCEDURE OUTLINE}

In [24], a method of complex transformation is proposed, which enables orthogonal complex filters with a canonic number of elements to be obtained. In accordance with this method, when the variable $z$ in an $N$-order real coefficient digital transfer function $H_{R}(z)$ is substituted by:

$$
z^{-1}=z^{-1} e^{j \frac{\pi}{2}}=j z^{-1},
$$

the complex coefficient orthogonal transfer function $H_{C}(z)$ will be obtained. It has alternately changing coefficients, i.e., real and imaginary, and can be easily represented by two $2 \mathrm{~N}$-order real coefficient transfer functions:

$$
H_{R}(z) \stackrel{z^{-1}=j z^{-1}}{\longrightarrow} H_{C}(z)=H_{R 1}(z)+j H_{R 2}(z) .
$$

If $H_{R}(z)$ is low-pass (LP), $H_{R 1}(z)$ and $H_{R 2}(z)$ will both be of band-pass (BP) type. However, a high-pass (HP) $H_{R}(z)$ will produce both BP and band-stop (BS) transfer functions.

Having two inputs and two outputs, an orthogonal complex filter is able to realise four real coefficients transfer functions at its different outputs, which are equal in couples as is apparent from Fig. 1.

The orthogonal complex transformation in (10), with the vector unit $i$ added to it and applied on a complex-coefficient orthogonal transfer function $H_{C}(z)$, will result in an orthogonal bicomplex transfer function:

$$
H_{C}(z) \stackrel{z^{-1}=i j z^{-1}}{\longrightarrow} H_{b i C}(z) .
$$

An $N$-order bicomplex orthogonal transfer function $H_{b i C}(z)$ can be represented by its scalar part $H_{S}(z)$ and vector part $H_{V}(z)$, both of these being complex coefficient functions of $2 \mathrm{~N}$-order. Additionally, $H_{b i C}(z)$ can be also represented via four realcoefficient transfer functions $H_{i}(z), i=1 \div 4$ of $4 N$-order, which compose the scalar and vector parts:

$$
\begin{aligned}
& H_{b i C}(z)=H_{S}(z)+i H_{V}(z)= \\
& =\left[H_{1}(z)+j H_{2}(z)\right]+i\left[H_{3}(z)+j H_{4}(z)\right]= \\
& =H_{1}(z)+j H_{2}(z)+i H_{3}(z)+k H_{4}(z) .
\end{aligned}
$$




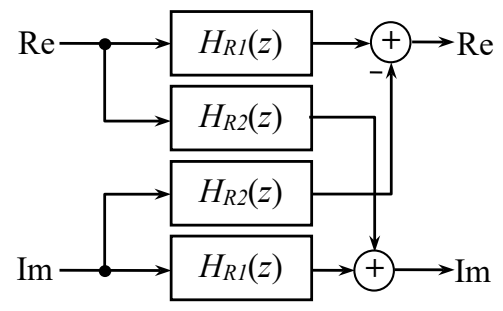

Fig. 1. Block diagram of a complex filter [3].

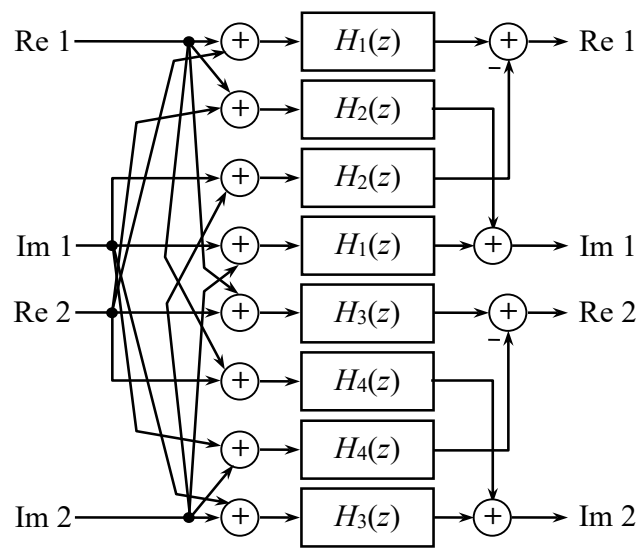

Fig. 2. Block diagram of a bicomplex filter.

In accordance with bicomplex number arithmetic, the scalar $H_{S}(z)$ and vector $H_{V}(z)$ parts can be derived as follows:

$$
\begin{aligned}
& H_{S}(z)=\frac{1}{2}\left[H_{b i C}(z)+H_{b i C}^{+}(z)\right] ; \\
& H_{V}(z)=\frac{1}{2 i}\left[H_{b i C}(z)-H_{b i C}^{+}(z)\right] .
\end{aligned}
$$

Applying the mathematical rules of complex functions, the real and imaginary parts of $H_{S}(z)$ and $H_{V}(z)$, i.e., $H_{i}(z)$ $(i=1 \div 4)$, can be determined to be:

$$
\left\{\begin{array}{l}
H_{1}(z)=\frac{1}{2}\left[H_{S}(z)+H_{S}^{*}(z)\right] ; \\
H_{2}(z)=\frac{1}{2 j}\left[H_{S}(z)-H_{S}^{*}(z)\right] ; \\
H_{3}(z)=\frac{1}{2}\left[H_{V}(z)+H_{V}^{*}(z)\right] ; \\
H_{4}(z)=\frac{1}{2 j}\left[H_{V}(z)-H_{V}^{*}(z)\right] .
\end{array}\right.
$$

In Fig. 2, a block diagram of an orthogonal bicomplex filter is shown. Having four inputs and four outputs, it will have sixteen fourth-order real-coefficient transfer functions altogether, four by four equal with \pm signs.

The design procedure thus presented extends a complex orthogonal digital filter to a bicomplex one. It is then straightforward to directly accomplish the circuit transformation of a real coefficient transfer function into a bicomplex orthogonal one:

$$
H_{R}(z) \stackrel{z^{-1}=i\left(z^{-1}+j z^{-1}\right)}{\longrightarrow} H_{b i C}(z) .
$$

However, instead of using the direct transformation (16) in this work we will first derive a complex orthogonal digital transfer function starting from a real coefficient one and then design the corresponding bicomplex orthogonal digital transfer function. This approach enables issues such as the canonicity and sensitivity of the three structures - real, complex and bicomplex - to be compared and contrasted.

\section{BILINEAR ORTHOGONAL BICOMPLEX FILTER DERIVATION}

Two of the best well-investigated canonic, low-sensitivity, free of limit cycles, bilinear real coefficient filters, named MHNS and LS11 [25], were selected and investigated

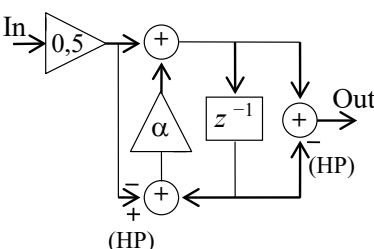

(a)

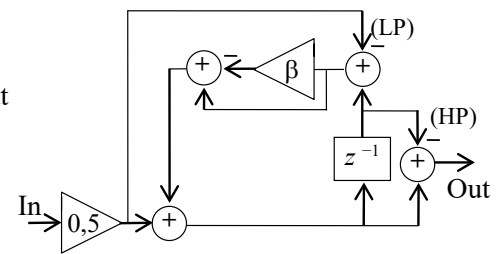

(b)
Fig. 3. Bilinear real sections: (a) MHNS-based; (b) LS11-based [25].

in this publication. Both are universal real digital filters, i.e., simultaneously realising LP and HP outputs (see Fig. 3). Their magnitude responses are unity for DC, and Nyquist's frequency in the case of LP and HP transfer functions, respectively, thus providing zero magnitude sensitivity. Since the experimental results for the HP outputs of both filters are similar, only those for the LP real transfer functions are discussed in the present study.

After the transformation (10) is applied to the LP real coefficient transfer functions, the orthogonal complex transfer functions are obtained and then represented by their real and imaginary parts. For the $M H N S$ filter this is:

$$
\begin{aligned}
H_{R}^{M H N S}(z) & =\frac{1-\alpha}{2} \frac{1+z^{-1}}{1-\alpha z^{-1}} \stackrel{z^{-1}=j z^{-1}}{\longrightarrow} \frac{1-\alpha}{2} \frac{1+j z^{-1}}{1-\alpha j z^{-1}}= \\
& =H_{C}^{M H N S}(z)=H_{R 1}^{M H N S}(z)+j H_{R 2}^{M H N S}(z)= \\
& =\frac{(1-\alpha)}{2} \frac{1-\alpha z^{-2}}{1+\alpha^{2} z^{-2}}+j \frac{\left(1-\alpha^{2}\right)}{2} \frac{z^{-1}}{1+\alpha^{2} z^{-2}},
\end{aligned}
$$

and for the $L S 11$ filter:

$$
\begin{aligned}
H_{R}^{L S 11}(z) & =\frac{\beta}{2} \frac{1+z^{-1}}{1-(1-\beta) z^{-1}} \stackrel{z^{-1}=j z^{-1}}{\longrightarrow} \frac{\beta}{2} \frac{1+j z^{-1}}{1-(1-\beta) j z^{-1}}= \\
& =H_{C}^{L S 11}(z)=H_{R 1}^{L S 11}(z)+j H_{R 2}^{L S 11}(z)= \\
& =\frac{\beta}{2} \frac{1-(1-\beta) z^{-2}}{1+(1-\beta)^{2} z^{-2}}+j \frac{\beta}{2} \frac{(2-\beta) z^{-1}}{1+(1-\beta)^{2} z^{-2}} .
\end{aligned}
$$

However, instead of using the direct transformation (16), in this study we will first derive a complex orthogonal digital transfer function starting from a real coefficient one and then design the corresponding bicomplex orthogonal digital transfer function. This approach enables issues such as the canonicity and sensitivity of the three structures - real, complex and bicomplex - to be compared and contrasted.

The bilinear orthogonal complex MHNS- and LS11-based filter structures are shown in Fig. 4 and Fig. 5, respectively. 


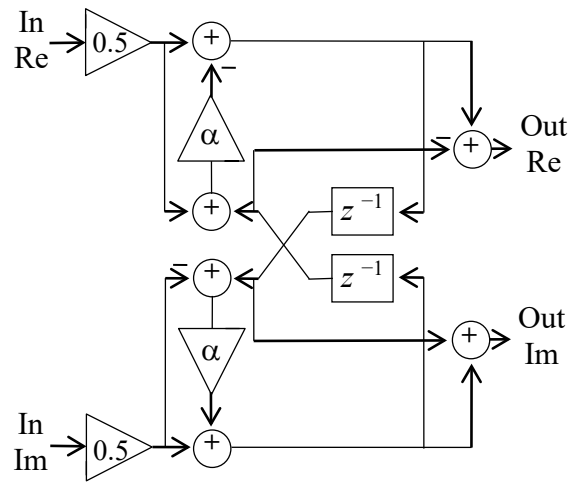

Fig. 4. Bilinear orthogonal complex MHNS-based filter [25].

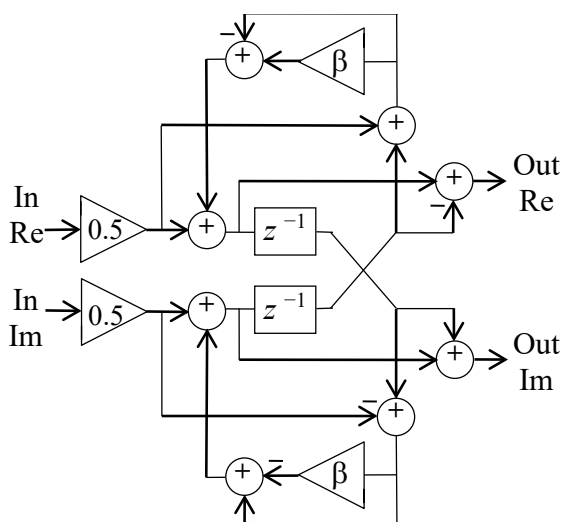

Fig. 5. Bilinear orthogonal complex $L S 11$-based filter [25].

The bicomplex orthogonal transformation (12) proposed in Section III, applied to the complex orthogonal transfer functions, (17) and (18), will result in bicomplex orthogonal transfer functions, which can be represented by their scalar and vector parts (13). For the MHNS-based filter (Fig. 6) this is as follows:

$$
\begin{aligned}
& H_{b i C}^{M H N S}(z)=H_{S}^{M H N S}(z)+i H_{V}^{M H N S}(z)= \\
& =\left[H_{1}^{M H N S}(z)+j H_{2}^{M H N S}(z)\right]+i\left[H_{3}^{M H N S}(z)+j H_{4}^{M H N S}(z)\right] .
\end{aligned}
$$

The four fourth-order real coefficients orthogonal transfer functions are as follows:

$$
\begin{aligned}
& H_{1}^{M H N S}(z)=\frac{1-\alpha}{2} \frac{1+\alpha^{3} z^{-4}}{1-\alpha^{4} z^{-4}} ; \\
& H_{2}^{M H N S}(z)=\frac{\alpha\left(1-\alpha^{2}\right)}{2} \frac{z^{-2}}{1-\alpha^{4} z^{-4}} ; \\
& H_{3}^{M H N S}(z)=\frac{\alpha^{2}\left(\alpha^{2}-1\right)}{2} \frac{z^{-3}}{1-\alpha^{4} z^{-4}} ; \\
& H_{4}^{M H N S}(z)=\frac{\alpha^{2}-1}{2} \frac{z^{-1}}{1-\alpha^{4} z^{-4}} .
\end{aligned}
$$

The same approach, when applied to the LS11-based filter (Fig. 7), produces the scalar and vector parts of the bicomplex orthogonal transfer function:

$$
\begin{aligned}
& H_{b i C}^{L S 11}(z)=H_{S}^{L S 11}(z)+i H_{V}^{L S 11}(z)= \\
& =\left[H_{1}^{L S 11}(z)+j H_{2}^{L S 11}(z)\right]+\left[H_{3}^{L S 11}(z)+j H_{4}^{L S 11}(z)\right],
\end{aligned}
$$

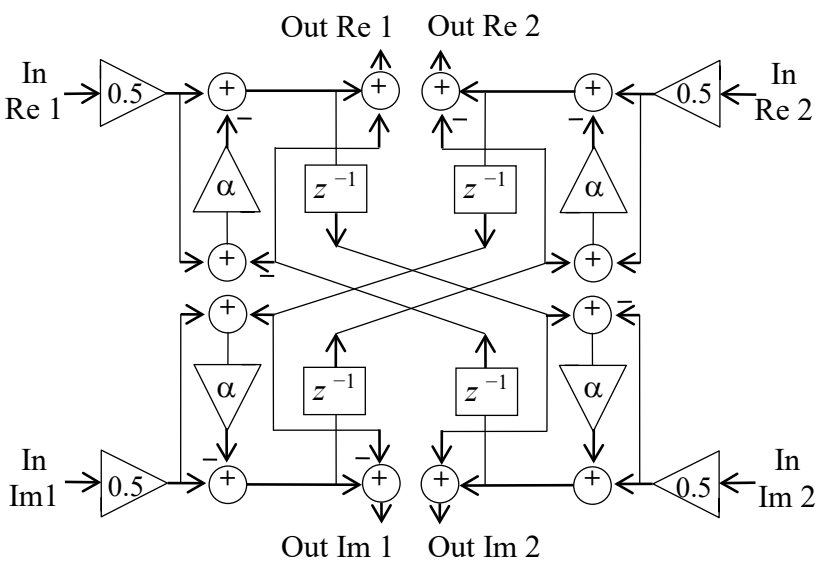

Fig. 6 Bilinear orthogonal bicomplex MHNS-based filter.

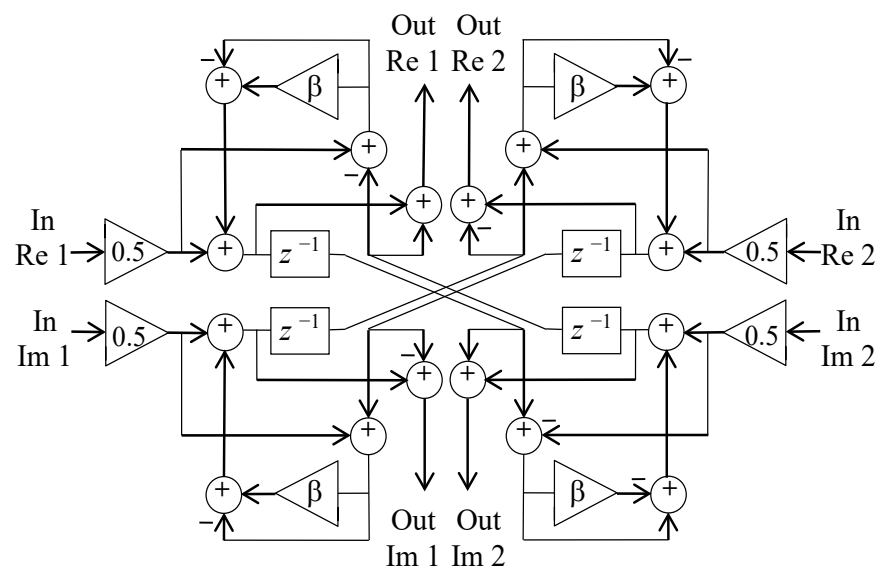

Fig. 7. Bilinear orthogonal bicomplex $L S 11$-based filter.

and their components - the LS11-based real coefficients orthogonal transfer functions:

$$
\begin{aligned}
& H_{1}^{L S 11}(z)=\frac{\beta}{2} \frac{1+(1-\beta)^{3} z^{-4}}{1-(1-\beta)^{4} z^{-4}} ; \\
& H_{2}^{L S 11}(z)=\frac{1-\beta}{2} \frac{\left[1-(1-\beta)^{2}\right] z^{-2}}{1-(1-\beta)^{4} z^{-4}} ; \\
& H_{3}^{L S 11}(z)=\frac{(1-\beta)^{2}}{2} \frac{\left[(1-\beta)^{2}-1\right] z^{-3}}{1-(1-\beta)^{4} z^{-4}} ; \\
& H_{4}^{L S 11}(z)=\frac{(1-\beta)^{2}-1}{2} \frac{z^{-1}}{1-(1-\beta)^{4} z^{-4}} .
\end{aligned}
$$

The bicomplex functions' constituents thus obtained will be examined for their sensitivity to quantizing the coefficients to different word-lengths.

\section{SENSITIVITY INVESTIGATION}

In this section, the coefficient sensitivity of all three types of filter (real, complex and bicomplex) will be discussed. Since narrow-band filtering is the most likely to be used in practice, the orthogonal filters considered in this study become narrowband for $\alpha=0.99(\beta=0.01)$. Canonic Sign-Digit (SD) code 
representation and fixed-point arithmetic were used and the word-length was changed from infinite (ideal case) to 3 bits.

The real LP prototype transfer functions were investigated under these conditions. It was shown that the coefficient sensitivity of the LS11 LP real bilinear filter is about one hundred times lower than that of the MHNS filter [25].

Another conclusion from the results of the experiments is that the low-sensitivity properties of the real circuits are directly acquired by the complex-coefficient orthogonal structures. The question is then whether this will also be the case for bicomplex orthogonal filters, and if they will inherit the levels of sensitivity to coefficient quantization of their complex orthogonal progenitor.

In order to answer this question, a number of computer simulations were conducted with respect to the bicomplex orthogonal transfer functions derived in the previous section. All real-coefficient transfer functions (20) for MHNS-based and (22) for $L S 11$-based were simulated for the same pole disposition near the unit circle. This resulted in the narrow-band orthogonal digital selective systems. Poles $p_{1,2}= \pm 0.99$ and $p_{3,4}= \pm j 0.99$ are achieved for $\alpha=0.99$ (MHNS-based) and $\beta=0.01$ (LS11-based) filters. In Figs. 8-11, experimental results for magnitudes of the LS11-based and MHNS-based orthogonal bicomplex transfer functions are shown for different coefficient word-lengths.

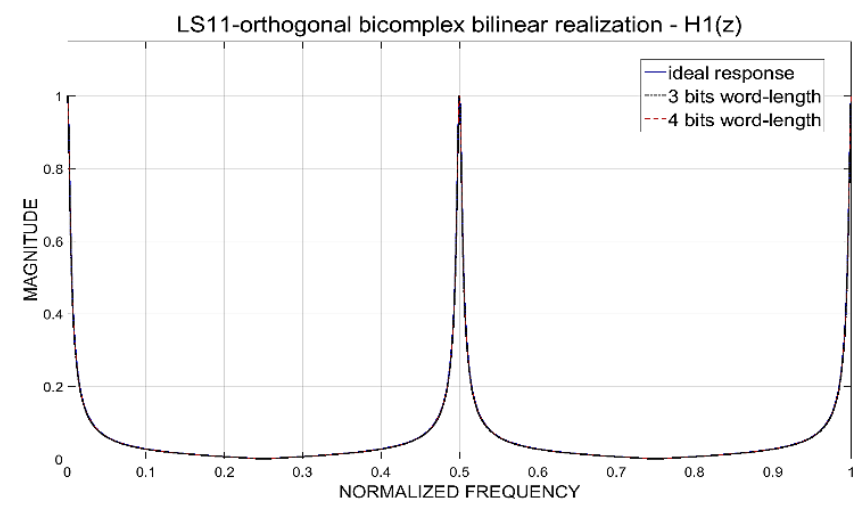

(a)

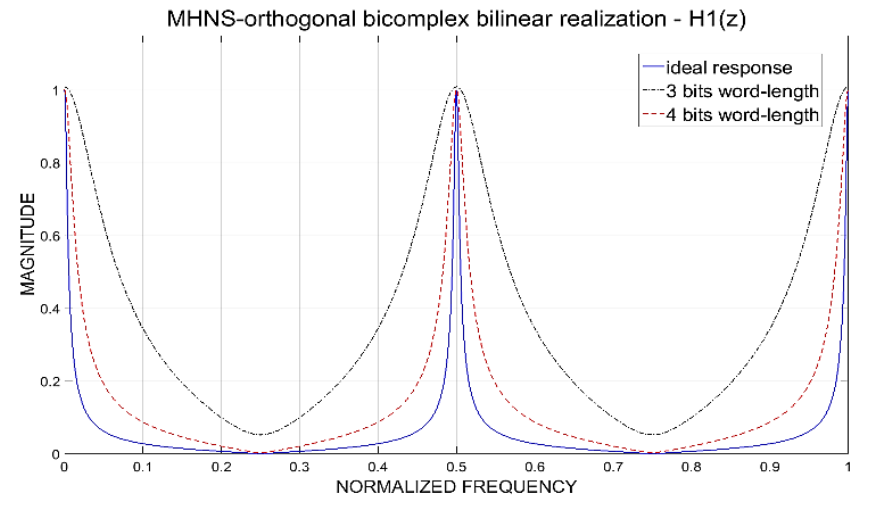

(b)

Fig. 8. Magnitude responses of the bicomplex orthogonal real-coefficient functions $H_{1}(z)$ for different word-lengths: (a) LS11-based; (b) MHNS-based.

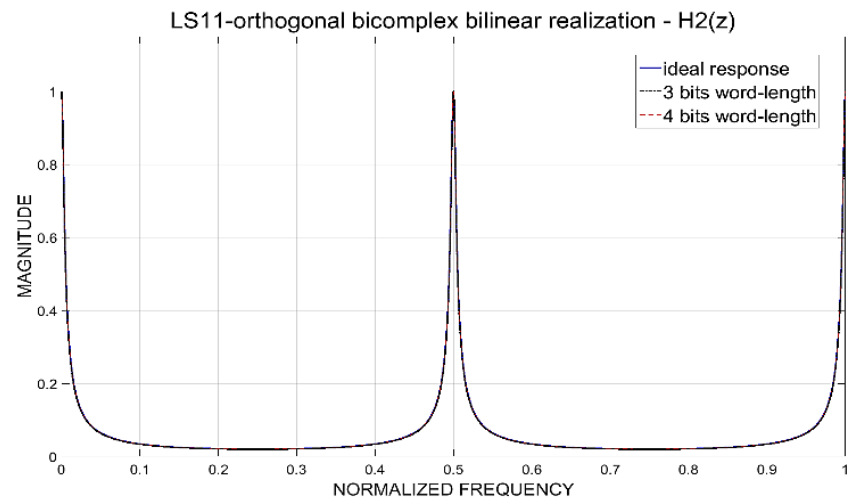

(a)

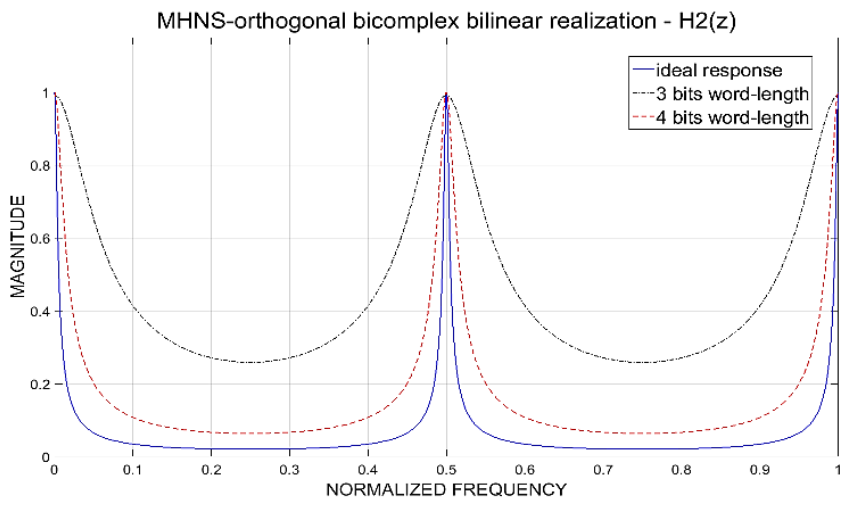

(b)

Fig. 9. Magnitude responses of the bicomplex orthogonal real-coefficient functions $\mathrm{H}_{2}(z)$ for different word-lengths: (a) LS11-based; (b) MHNS-based.

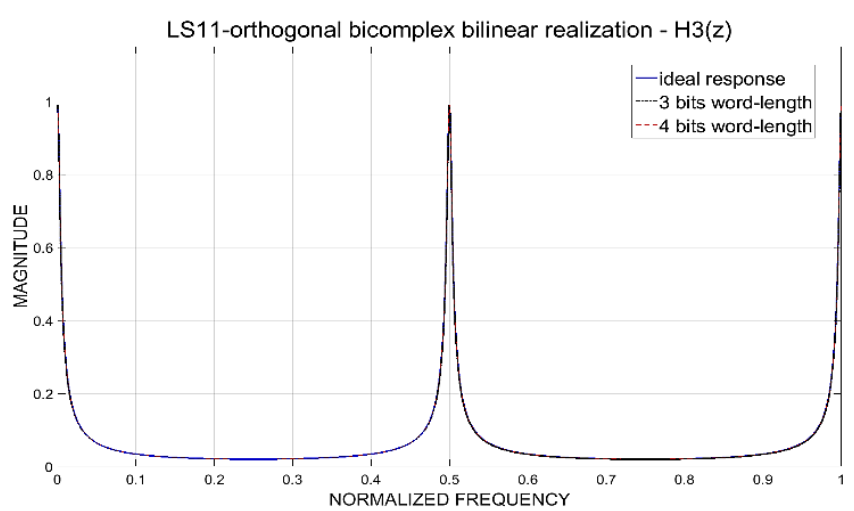

(a)

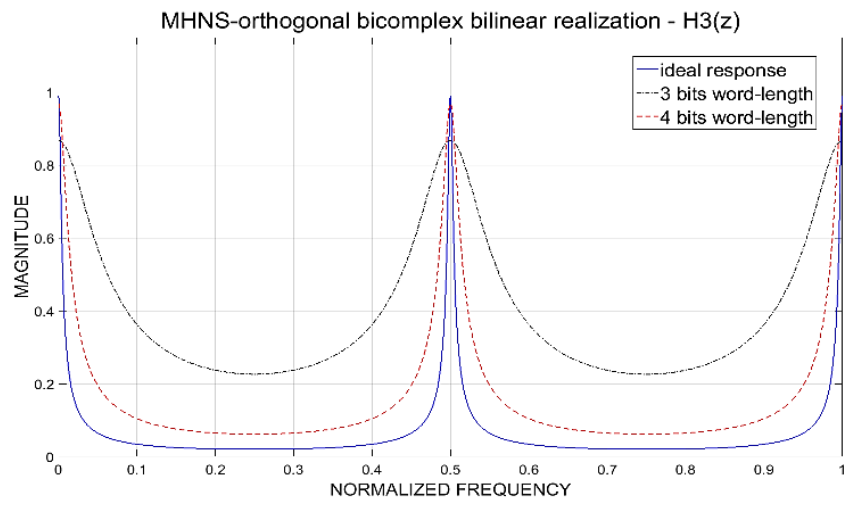

(b)

Fig. 10. Magnitude responses of the bicomplex orthogonal real-coefficient functions $\mathrm{H}_{3}(z)$ for different word-lengths: (a) LS11-based; (b) MHNS-based. 
It can be seen that the orthogonal LS11-based bicomplex structure has an almost ideal magnitude response, even when the word-length is reduced to only 3 bits. The magnitude response of the MHNS-structure is considerably changed when the wordlength is limited to 3 bits and the filter ceases to be narrow-band. At 4 bits the magnitude response degrades even more, further worsening the selectivity of the filter. At this word-length of the coefficient $\alpha$ problems are also observed with respect to the magnitude unity gain and these are most clearly manifested in magnitudes $|\mathrm{H} 3|$ (Fig. 10b) and $|\mathrm{H} 4|$ (Fig. 11b). The magnitude response attains a maximum value of only 0.8672 for $|\mathrm{H} 3|$, while for $|\mathrm{H} 4|$ the unity gain is exceeded and reaches 1.133 . The experiments for the LS11 bicomplex orthogonal algorithm, conducted under the same conditions, indicate preservation of the unity gain, as seen in the graphs of Fig. 8a, Fig. 9a, Fig. 10a, and Fig. 11a. The exact values differ from unity very slightly, but are within the anticipated computational error.

\section{CONCLUSIONS}

This paper outlines a method of designing efficient orthogonal bicomplex DSP algorithms, commencing from a

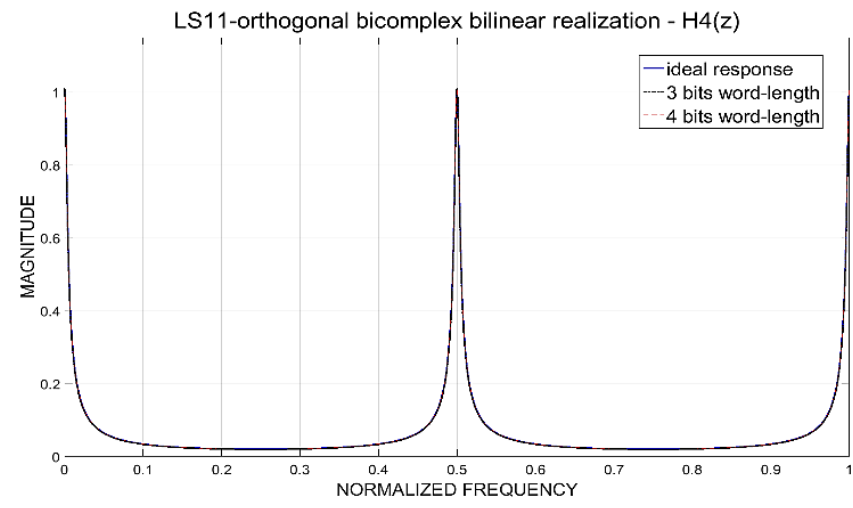

(a)

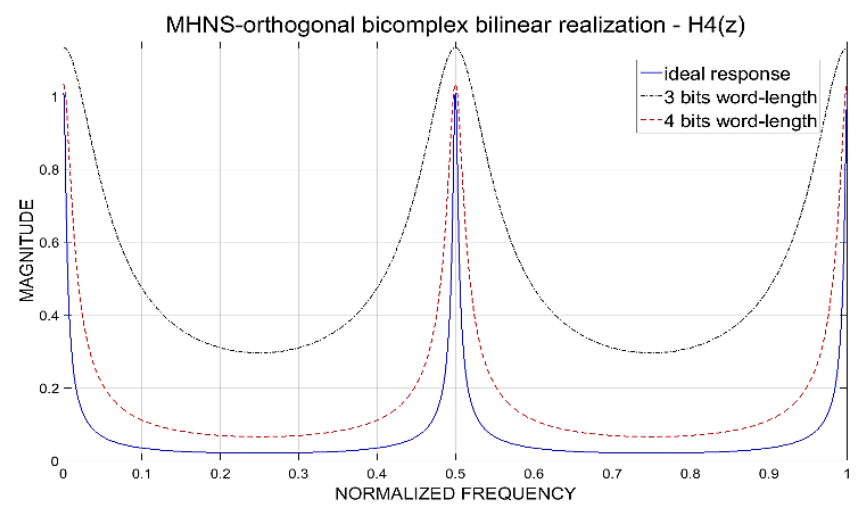

(b)

Fig. 11. Magnitude responses of the bicomplex orthogonal real-coefficient functions $H_{4}(z)$ for different word-lengths: (a) $L S 11$-based; (b) MHNS-based.

transfer function with complex or real coefficients. This novel method has universal applicability and is thus usable in the creation of canonical digital filters, regardless of their type or order.

Due to the practical importance of narrow-band orthogonal filters, this type of transfer function was developed and investigated for different coefficient word-lengths. Experimental demonstrations reveal only minor changes in the magnitude response of the LS11 low sensitivity bicomplex orthogonal filter, using only three bits of SD-code. However, MHNS filters show significant degradation in magnitude response if word-length is decreased to four bits. Moreover, the resulting bicomplex orthogonal functions inherit sensitivity properties of the real and complex transfer functions. Following the approach proposed will lead to the derivation and investigation of a multitude of novel efficient bicomplex orthogonal filters.

The presented method for the design of bicomplex orthogonal DSP algorithms is also appropriate for the derivation of second-order filters, which will provide cascade bicomplex orthogonal filter realisation of any order. Additionally, the possibility for simplification of the circuits and further parallelism will make them very attractive for telecommunications and other DSP applications and will ensure a considerable reduction in the complexity and cost of the equipment.

A subject of future research might be the derivation of a generalized bicomplex transformation, which would make parallel processing of any pair of non-orthogonal complex signals possible. The use of new non-orthogonal waveforms is a promising advanced transmission method that will allow for the efficient allocation of wireless interface resources in modern telecommunications networks. Effective bicomplex filters can be a powerful tool for processing non-orthogonal waveforms as both single selective DSP algorithms and as building blocks of more sophisticated parallel digital processing systems, such as filter banks.

\section{ACKNOWLEDGMENT}

The research was supported by the research project KP-06N27 / 3 / 08.12.2018 "Resource Self-configuration and Management in Ultra-dense Networks with User Centric Wireless Access" of the Bulgarian Research Fund of the Ministry of Education and Science.

\section{REFERENCES}

[1] W. R. Hamilton, "Elements of Quaternions," New York, DC, USA: Chelsea Publishing Company, 1969.

[2] H. D. Schutte and J. Wenzel, "Hypercomplex numbers in digital signal processing”, Proc. IEEE Singapore International Symposium on Circuits and Systems, pp. 1557-1560, 1990.

[3] Z. Nikolova, G. Stoyanov, G. Iliev, and V. Poulkov, “Complex coefficient IIR digital filters," Chapter 9 in Digital Filters, F. P. G. Márquez, Ed., InTechOpen, 2011, pp. 209-239. https://doi.org/10.5772/648

[4] T. Bulow and G. Sommer, "Hypercomplex signals - a novel extension of the analytic signals to the multidimensional case", IEEE Transaction on Signal Processing, vol. 49, no. 11, Nov. 2001, pp. 2844-2852. https://doi.org/10.1109/78.960432

[5] A. Khatabi, A. Tmiri, and A. Serhir, "Quaternion angular radial transform and properties transformation for color-based object recognition", Pattern Recognition and Image Analysis, vol. 26, no. 4, 1 Oct. 2016, pp. 705-713. https://doi.org/10.1134/S1054661816040064

[6] V. G. Labunets, E. V. Labunets, K. Egazarian and J. Astola, "Hypercomplex moments application in invariant image recognition", Proc. of International Conference on Image Processing (ICIP98), Chicago, USA, 1998, vol. 2, pp. 257-261. 
[7] W. K. Wong, G. C. Lee, C. K. Loo and R. Lock, "Quaternion based fuzzy neural network classifier for MPIK dataset's view-invariant color face image recognition", Informatica (Slovenia), vol. 37, no. 2, 2013, pp. 181192.

[8] C. Evans, S. J. Sangwine, and T. A. Ell, "Hypercomplex color-sensitive smoothing filters", Proc. of International Conference of Image Processing, vol. 1, pp. 541-544, 2000.

[9] F. Tao, and W. Qian, "Image hash authentication algorithm for orthogonal moments of fractional order chaotic scrambling coupling hyper-complex number”, Measurement, vol. 134, Feb. 2019, pp. 866-873. https://doi.org/10.1016/j.measurement.2018.11.079

[10] C. E. Moxey, S. J. Sangwine, and T. A. Ell, "Color-grayscale image registration using hypercomplex phase correlation", Proc. of International Conference on Image Processing, vol. 2, pp. II-385-II-388, 22-25 Sept. 2002

[11] S. J. Sangwine and T. A. Ell, "Hypercomplex auto- and cross-correlation of color images", Proceedings of IEEE International Conference on Image Processing (ICIP99), 24-28 Oct. 1999, Kobe, Japan, pp. 319-322.

[12] S. J. Sangwine et al., "Color image filters based on hypercomplex convolution", IEEE Proc. Vis. Image Signal Processing, vol. 147, no. 2 , pp. 89-93, April 2000. https://doi.org/10.1049/ip-vis:20000211

[13] L. Lu, X. Zhang, and X. Xu, "Hypercomplex extreme learning machine with its application in multispectral palmprint recognition", PLOS ONE, vol. 14, no. 4, April 2019. https://doi.org/10.1371/journal.pone.0209083

[14] V. Dimitrov, T. Cooklev and B. Donevsky, "On the multiplication of reduced biquaternion and applications". Information Processing Letters, vol. 43, no. 3, pp. 161-164, 1992. https://doi.org/10.1016/00200190(92)90009-K

[15] H. Toyoshima, "Computationally Efficient implementation of hypercomplex digital filters", IEICE Trans. Fundamentals, vol. E85-A, no. 8, pp. 1870-1876, August 2002.

[16] K. Ueda and S.-I. Takahashi, "Strictly proper digital filters with hypercomplex coefficients", Proc. ECCTD'93 European Conference on Circuit Theory \& Design, pp. 739-744, Sept. 1993.

[17] K. Ueda, K. Mazukami, and S.-I. Takahashi, "Realization of complex coefficient digital filters based on hypercomplex arithmetic", Proc. European Signal Processing Conference (EUROSIPCO’94), pp. 363 366, Sept. 1994.

[18] H. Osaco, K. Ueda, and S.-I. Takahashi, "Digital filter with eight elements hypercomplex coefficient", Proc. of 12th European Conference on Circuit Theory \& Design (ECCTD'95), ITU, Istanbul Technical University, Istanbul, Turkey, August 1995, pp. 659-662.

[19] D. Alfsmann, and H. G. Göckler, "Hypercomplex Bark-scale filter bank design based on allpass-phase specifications", European Signal Processing Conference, EUSIPCO 2012, Bucharest, Romania, 27-31 August 2012, pp. 519-523.

[20] Y. N. Li, "Quaternion polar harmonic transforms for color images", IEEE Signal Processing Letters, vol. 20, no. 8, 2013, 6530686, pp. 803-806. https://doi.org/10.1109/LSP.2013.2267775

[21] B. Hu, Y. Zhou, L.-D. Li, J.-Y. Zhang and J.-S. Pan, "Polar linear canonical transform in quaternion domain", Journal of Information Hiding and Multimedia Signal Processing, vol. 6, no. 6, 2015, pp. 11851193.

[22] M. Kamata, and S.-I. Takahashi, "Orthogonal filter with hypercomplex coefficients, including cases of complex and real ones", Proc. of ECCTD'97, Budapest, Hungary, Sept. 1997, pp. 594-598.

[23] M. Okuda, M. Kamata, and S.-I. Takahashi, "Realization of an orthogonal filter with hypercomplex coefficients", Electronics \& Communications in Japan, Part III: Fundamental Electronic Science, vol. 85, no. 3, pp. 5260, 2002. https://doi.org/10.1002/ecjc. 1079

[24] E. Watanabe and A. Nishihara, "A synthesis of a class of complex digital filters based on circuitry transformations". IEICE Trans., vol. E-74, no. 11, pp. 3622-3624, Nov. 1991.

[25] G. Stoyanov, M. Kavamata, and Z. Valkova. "New First and SecondOrder Very Low-Sensitivity Bandpass/Bandstop Complex Digital Filter Sections", Proc. IEEE 1997 Region 10 th Annual Conf., TENCON'97, Brisbane, Australia, vol. 1, pp. 61-64, Dec. 2-4, 1997.

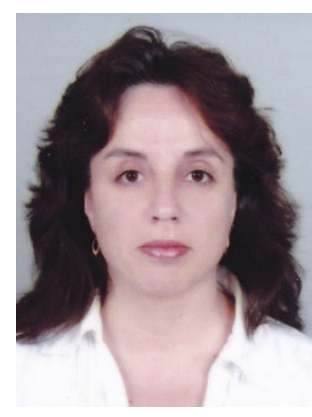

Zlatka Valkova-Jarvis was awarded an M. Sc. degree in Electrical Engineering and $\mathrm{Ph}$. D. degree in Theoretical Fundamentals of Telecommunications by the Technical University of Sofia (TUS), Bulgaria. Her main research interests include digital signal processing, communication circuits, and physical layer security in telecommunications. In these research areas she has over 70 papers and publications, and is author and co-author of numerous textbooks, manuals and book-chapters. She has participated in a multitude of research projects in different areas of telecommunications, and has been a visiting researcher/lecturer in several European universities. Currently, Ms Jarvis is an Associate Professor in the Department of Communication Networks, Faculty of Telecommunications, TUS, and is also the Vice-Dean of the Faculty.

Technical University of Sofia, 8 Kliment Ohridsky Blvd, Sofia 1000, Bulgaria. E-mail: zvv@tu-sofia.bg

ORCID iD: https://orcid.org/0000-0002-6627-0148

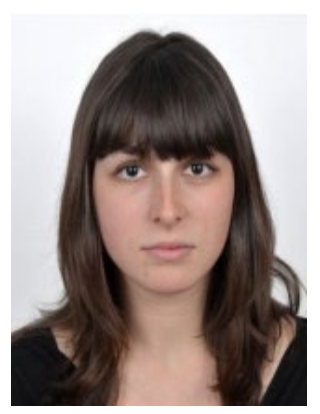

Dimitriya Mihaylova attained B. Sc. and M. Sc. degrees in Telecommunications at the Technical University of Sofia (TUS), Bulgaria, in 2014 and 2016. She completed her B. Sc. thesis "Development of a communication system for ArduCopter model through the Iridium Satellite Network" as an Erasmus student at the Universidad de Las Palmas de Gran Canaria, Spain. Her Master's thesis project, "Evaluation of the Dielectric Properties of Materials at High Temperatures", was developed at the Universitat Politècnica de València, Spain. In 2019 she gained a Ph. D. in Communication Networks and Systems from TUS. Her current research interests are in the field of her $\mathrm{PhD}$ thesis - Physical Layer Security techniques for the protection of wireless systems.

Since 2016 she has been with the Department of Communication Networks of TUS, now as an Assistant Professor.

Technical University of Sofia, 8 Kliment Ohridsky Blvd, Sofia 1000, Bulgaria. E-mail: dam@tu-sofia.bg

ORCID iD: https://orcid.org/0000-0002-6552-2139

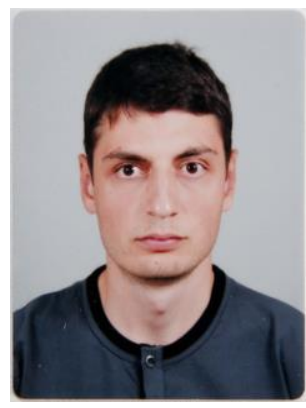

Viktor Stoynov received his B. Sc. and M. Sc. degrees in Telecommunications from the Technical University of Sofia (TUS), Bulgaria, in 2012 and 2014 respectively. In 2018 he obtained his $\mathrm{Ph} . \mathrm{D}$. in Theoretical Fundamentals of Telecommunications at the TUS on the topic of "Investigation of Resource Allocation in Abstract Modelled Indoor Wireless Communication Environments in New Generation Networks". He has participated in several nationally-funded research projects. His research interests are in the fields of interference management in heterogeneous and ultra-dense networks and intelligent resource allocation strategies, in which he has 18 papers and publications. At present, Mr Stoynov is an Assistant Professor in the Department of Communication Networks, Faculty of Telecommunications, TUS, Bulgaria.

Technical University of Sofia, 8 Kliment Ohridsky Blvd, Sofia 1000, Bulgaria. E-mail: vstoynov@tu-sofia.bg

ORCID iD: https://orcid.org/0000-0002-2624-9942 